\title{
3D Multiscale Characterization of Discontinuities in Underwater Wet Welds.
}

\author{
S. Paciornik ${ }^{1}$, L. F. Silva ${ }^{1}$, V. R. dos $\operatorname{Santos}^{1}$, N. Chawla ${ }^{2}$, G. Schneider ${ }^{3}$ and T. Bernthaler ${ }^{3}$ \\ 1. Chemical and Materials Engineering, PUC-Rio, Rio de Janeiro, Brazil. \\ 2. Materials Science and Engineering ASU, Tempe, USA. \\ 3. Materials Research Institute, Aalen University, Aalen, Germany.
}

Underwater wet welding (UWW) is a critical procedure for the repair of offshore structures, mainly related to oil production and transportation. The harsh environmental conditions in which the weld is performed has strong consequences to the structural reliability of the welded parts. High cooling rates due to direct contact with water and the presence of hydrogen derived from water dissociation leads to the formation of defects, such as pores and cracks in the weld metal (WM), which adversely affect mechanical properties. During cooling, weld beads contract both in transverse and longitudinal directions. It is well established that longitudinal contractions are responsible for higher residual stress after welding. Consequently, in wet welds, the low toughness associated with high hydrogen contents in the WM can lead to nucleation of cracks [1,2]. Inclusions also tend to form as the available oxygen reacts with various elements forming stable oxides that may be eliminated in the slag or get trapped within the weld metal as spherical shaped inclusions.

Welding depth relates to water pressure and partial pressures of oxygen and hydrogen. Thus porosity is directly dependent on welding depth. Oxide inclusion content tends to increase with welding depth but saturates as the solubility limit of oxygen reaches its peak value. Cracks depend on the availability of diffusible hydrogen, what depends on the specific type of electrode employed. For instance, rutillic electrodes tend to produce weldments with higher diffusible hydrogen in comparison to oxidizing electrodes. However, oxidizing electrodes tend to result in higher inclusions content, which is detrimental to the mechanical properties of the weld metal [3].

UWW is an excellent test case for multiscale analysis techniques due to the wide variation in the microstructural characteristics of pores, cracks and inclusions [4,5]. Discontinuity sizes range from $\mathrm{nm}$ (inclusions) to hundreds of $\mu \mathrm{m}$ (pores). Shape varies from spherical inclusions to elongated wormhole shaped pores and thin cracks. Pore spatial and orientation distribution is also complex, affected by the scape of gases from the liquid weld as it rapidly solidifies. Cracks show a predominant orientation transverse to the weld axis while inclusions are randomly distributed in the WM. These characteristics also highlight the relevance of 3D techniques, as traditional 2D microscopy would not be able to reveal these complex shapes and relationships. Thus, X-ray microtomography $(\mu \mathrm{CT})$ was employed to reveal pores and cracks while FIB/SEM was used to characterize inclusions.

Extensive image processing (noise filtering, image alignment, manual and automatic thresholding and post-processing) and 3D measurements (volume, shape) were performed with FIJI/ImageJ. Figure 1 shows 3D models for pores and cracks obtained from $\mu \mathrm{CT}$. The expected pore elongation and crack orientation are clearly visible. Figure 2 shows a 3D model for inclusions obtained with FIB/SEM, confirming the random distribution of mainly spherical objects. These results highlight the relevance of analyzing discontinuities in 3D, employing complementary techniques to span the vast scale range involved [6]. 
References:

[1] T. G. Gooch, Metal Construction 15 (1983), p 164.

[2] T. G. Gooch, Metal Construction 15 (1983), p 206.

[3] V. R. Santos et al, Welding Journal 91 (2012), p. 319.

[4] S PACIORNIK et al, Proceedings of the $1^{\text {st }}$ International Conference on 3D Materials Science (2012), p. 177.

[5] E PADILLA et al, Materials Characterization, 83 (2013), p. 139.

6 The authors acknowledge funding from CNPq, FAPERJ and CAPES, Brazilian funding agencies.

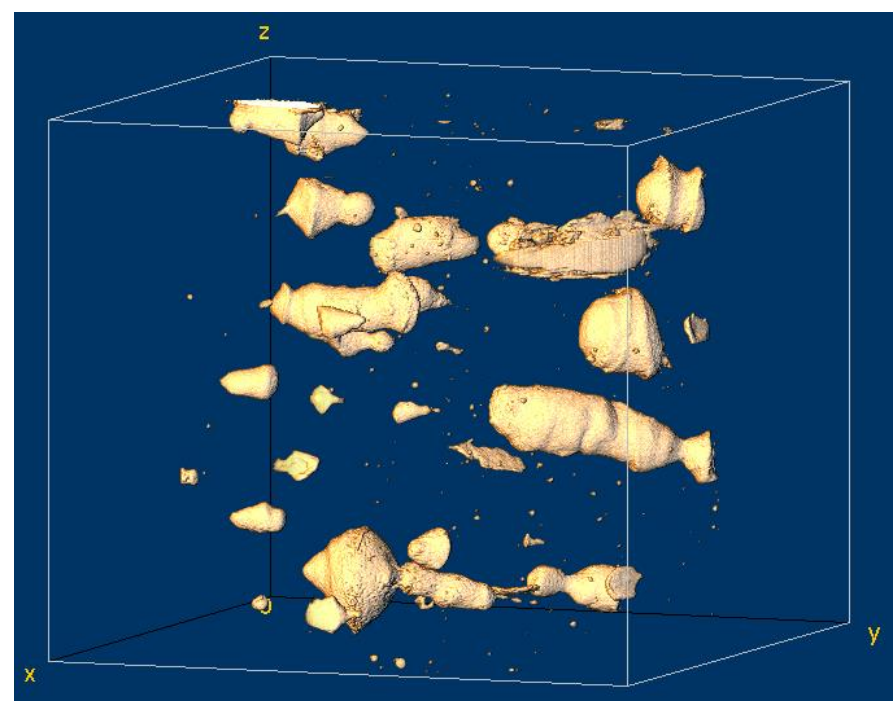

$1000 \mu \mathrm{m}$

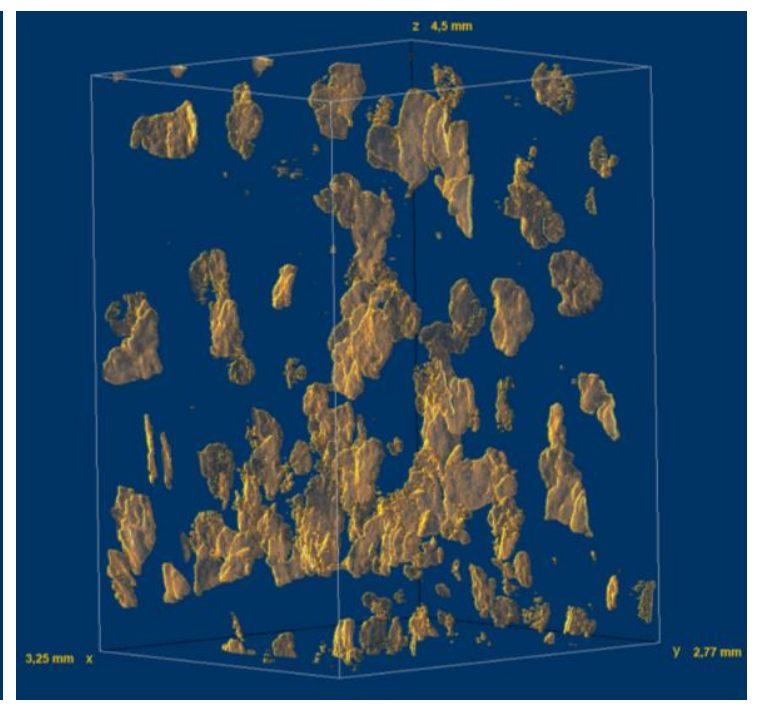

$1000 \mu \mathrm{m}$

Figure 1. 3D models obtained from $\mu \mathrm{CT}$. Pores (left), cracks (right).

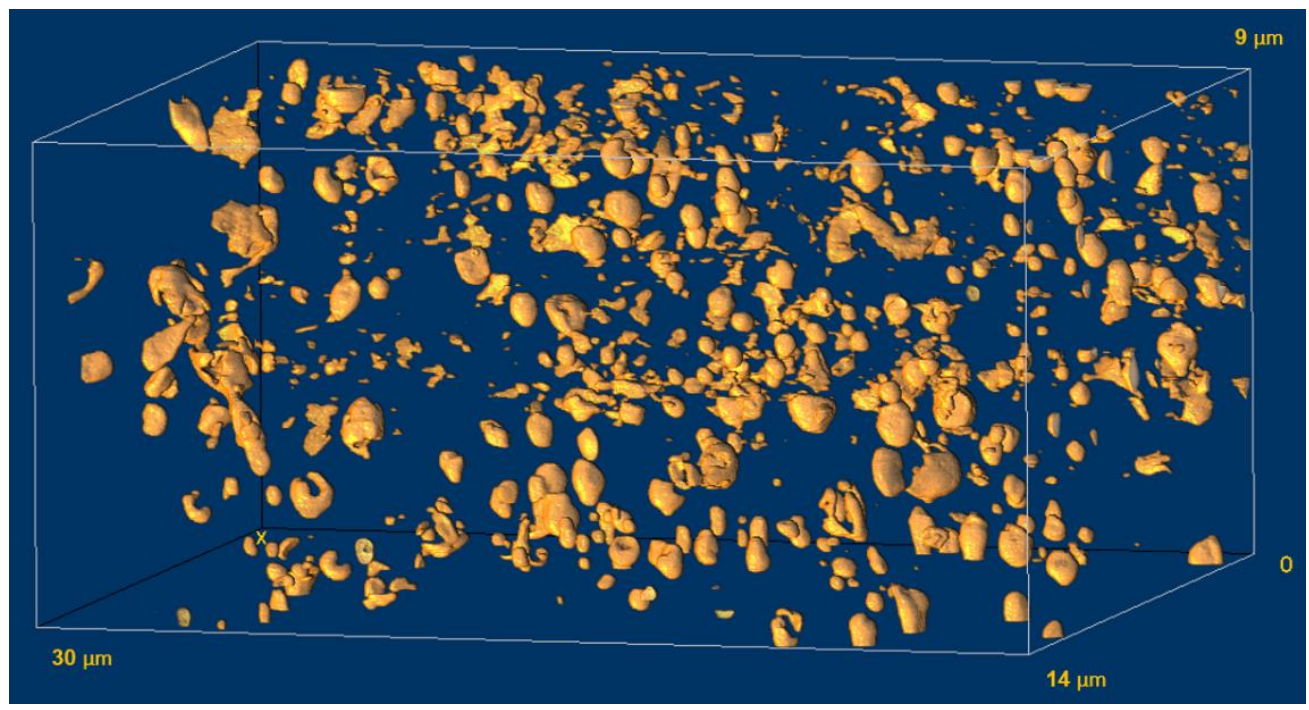

$3 \mu \mathrm{m}$

Figure 2. 3D model of inclusions, obtained from FIB/SEM. 